\title{
Aortenklappenstenose
}

\section{Zehn Jahre nach der ersten TAVI-Implantation: Bilanz überzeugt}

Zehn Jahre nach der ersten Implantation einer Transkatheter-Aortenklappe (TAVI) liegt eine positive Bilanz vor. Prof. Alain Cribier aus Rouen (Frankreich) hatte die erste Prozedur bei einem schwerkranken Patienten mit Aortenklappeninsuffizienz im Jahr 2002 vorgenommen. Seitdem wurden weltweit etwa 40000 TAVI-Prozeduren durchgeführt.

Die derzeit geltenden Leitlinien von ESC sowie von ACCF, AATS, SCAI und STS raten zu einer TAVI bei Hochrisikopatienten mit schwerer Symptomatik und einem EuroRisiko-Score von $\geq 20 \%$. Eine TAVI sollte nicht vorgenommen werden, wenn der Patient ausschließlich einen herzchirurgischen Eingriff aus persönlicher Neigung ablehnt. Außerdem sollte die Lebenserwartung des Patienten mehr als ein Jahr betragen. Prof. Helmut Baumgartner aus München erklärte: „Wir bewegen uns bei der Indikation in Richtung Patienten mit geringerem Risiko." Das Problem bestehe derzeit noch darin, die ge- naue Indikation auf Grundlage der Studien in Leitlinien zu definieren. Vorteil des Transkatheter-Verfahrens bei schwerkranken Patienten, sei, dass die Brust und das Herz nicht geöffnet werden müssen, kein Herzstillstand induziert wird und keine extrakorporale Zirkulation eingerichtet werden müssen.

Aus den Registern zur Edwards-SapienTranskatheter-Klappe in Frankreich, Belgien und Kanada geht hervor, dass die 30-TagesMortalität unter $10 \%$ liegt. Die 1-JahresMortalität im SOURCE-Register lag bei 18,9\%. Die Rate an Schlaganfällen in Verbindung mit der Prozedur liegt in den Registern bei $3-5 \%$.

Eine Metaanalyse von 48 Studien mit 13216 Patienten über 80 Jahren hat kürzlich ergeben, dass die unmittelbare postoperative Mortalität bei $6,7 \%$ liegt, das 1-JahresÜberleben bei $88 \%$, das 3-Jahres-Überleben bei $79 \%$ und das 5-Jahres-Überleben bei $65 \%$.

Diese Zahlen werden aber erst dann richtig bewertet, wenn die Lebenserwartung ohne Therapie bei diesen inoperablen schwer herzkranken Patienten gegenübergestellt wird, erläuterte Prof. Alain Cribier im Gespräch mit SpringerMedizin. Der Direktvergleich zwischen Standard und TAVI wurde in der PARTNER-US-Studie untersucht, wo nach zwölf Monaten eine Mortalitätsreduktion von $50,7 \%$ unter der Standardtherapie auf 30,7\% den großen Überlebensvorteil durch TAVI deutlich machte. Später folgten die PARTNERStudien, die diese Tendenz bestätigten.

Mit derTAVI-Prozedur verbundene Komplikationen wie Regurgitationen werden mit der Wahl der richtigen Klappengröße umgangen. Wann die 23-mm-Klappe, die 26-mm-Klappe oder die inzwischen verfügbare 29-mm-Klappe verwendet werden soll, darüber liegen bereits Erfahrungen vor. Ausgemessen wird der oftmals exzentrische Annulus heute am besten mit der kardialen MRT, schlug Prof. Stephan Achenbach aus Gießen vor. Cribier selbst empfahl ein Ausmessen mit dem Katheter.

(rk) I

Satellitensymposium, 78. Jahrestagung der DGK, Mannheim, 12. April 2012 (Veranstalter: Edwards Life Science)

\section{Herzinsuffizienz}

\section{Schlechtere Prognose bei Eisenmangel}

Ein Eisenmangel oder eine Anämie können bei Patienten mit chronischer Herzinsuffizienz prognostisch relevant sein. Daher sollte bei Patienten mit Herzinsuffizienz der Eisenstatus erhoben werden. Dies betont die europäische Gesellschaft für Kardiologie (ESC) in ihren aktuellen Leitlinien.

Eine große, multizentrische Registerstudie in Deutschland bei 1278 Patienten mit chronischer Herzinsuffizienz ergab, dass 22,4\% der Patienten eine manifeste Anämie mit einem Hämoglobin-Wert $<12 \mathrm{~g} / \mathrm{dl}$ aufweisen. Herzinsuffiziente Patienten mit Anämie hatten im Vergleich zu nicht anämischen eine mehr als doppelt so hohe 1-Jahres-Mortalität (23,1 vs. $10,9 \% ; p<0,001)$. Auch die Liegezeiten im Krankenhaus waren länger ( 11 vs. 8 Tage; $p<0,001$ ). Die Registerdaten belegen außerdem, dass eine Anämie bei Patienten mit Herzinsuffizienz ein eigenständiger Risikofaktor ist.

In 79\% der Fälle liegt der Anämie bei Herzinsuffizienz ein Eisenmangel zugrunde. Für die Korrektur eines Eisenmangels bei diesen Patienten empfiehlt die ESC eine intravenöse Eisensupplementierung. Die FAIRHF-Studie ergab, dass eine intravenöse Therapie mit Eisencarboxymaltose (ferinject ${ }^{\oplus}$ ) innerhalb von vier Wochen die Symptome, die NYHA-Klasse und die Lebensqualität von Patienten mit chronischer Herzinsuffizienz und Eisenmangel signifikant im Vergleich zu Placebo verbessert. Die Effekte waren unabhängig vom gleichzeitigen Vorliegen einer Anämie.

(red) II

Nach Informationen von Vifor 